\title{
TRAUMATISMO TORÁCICO: ESTUDO RETROSPECTIVO DE 168 CASOS
}

\author{
THORACIC TRAUMA: RETROSPECTIVE STUDY OF 168 CASES
}

Ricardo Mauricio Batista Feitosa Cuba, ACBC-MA ${ }^{1}$; Jose Antonio Ferreira de Bezerra, TCBC-MA ${ }^{1}$

\begin{abstract}
RESUMO: Objetivo: Avaliar o atendimento ao paciente portador de traumatismo torácico. Método: Estudo retrospectivo de 168 casos de trauma do tórax, com ênfase na abordagem inicial, conduta operatória e cuidados pós-operatórios. Resultados: Dos 168 pacientes, 120 eram do sexo masculino e a média de idade encontrada foi de 35,5 anos. Dez pacientes foram toracotomizados de urgência, quatro por ferimento cardíaco, quatro devido à lesão de vasos pulmonares, um por lesão do saco pericárdico e outro do pedículo pulmonar. Os demais tiveram suas condições clínicas corrigidas através de simples drenagem torácica do hemitórax atingido. Ocorreram dois óbitos não cirúrgicos e um devido à insuficiência respiratória Conclusões: O traumatismo torácico, além de ser muito freqüente, é na maioria das vezes, de fácil resolução. Podem ocorrer, no entanto, lesões torácicas graves envolvendo órgãos vitais que merecem intervenção mais agressiva e perícia técnica, com suporte hospitalar de alto nível (Rev. Col. Bras. Cir. 2005; 32(2): 57-59).
\end{abstract}

Descritores: Trauma; Traumatismos torácicos; Tórax; Cuidados pós-operatórios.

\section{INTRODUÇÃO}

O trauma tem sido a principal causa de morte na população adulta jovem no mundo atual, e o traumatismo torácico tem se destacado em virtude de fatores vinculados ao avanço tecnológico, como meios de transporte cada vez mais velozes e pelo aumento da violência urbana ${ }^{1-3}$.

Embora, na maioria dos casos, um trauma envolvendo a caixa torácica tenha uma abordagem inicial e sistemática simples, pois dados da própria inspeção física já fornecem indícios diagnósticos, algumas vezes a pouca experiência ou inobservância de aspectos relevantes ao politraumatizado como um todo, pode comprometer seriamente a vida do paciente. Não é rara a concomitância de lesões traumáticas importantes de outros segmentos corporais como a cavidade abdominal ou craniana, que podem ser prioritárias no momento da admissão no pronto-socorro. Não obstante, o principal objetivo na abordagem inicial do paciente com o tórax lesado é manter a capacidade ventilatória dos pulmões e a prevenção da hipóxia. A avaliação clínica rigorosa, baseada nos princípios do suporte de vida avançado em trauma (ATLS) ${ }^{4}$ pode fazer uma grande diferença neste momento.

O trauma de tórax pode ser classificado em fechado ou penetrante, em função da abertura ou não da cavidade pleural. Qualquer um dos órgãos contidos na cavidade torácica pode ser atingido durante um trauma, dependendo de seu mecanismo, trajetória e magnitude. Órgãos abdominais também podem ser acometidos, como o fígado e o baço mais frequentemente, no trauma toraco-abdominal ${ }^{1}$.

O objetivo deste trabalho foi o de estudar o traumatismo torácico de pacientes tratados no Hospital Djalma Marques, da rede pública municipal de São Luís (MA)

\section{MÉTODO}

Estudo retrospectivo de pacientes vítimas de traumatismo torácico atendidos no Hospital Djalma Marques entre 2001 a 2002, através de ficha protocolo padronizada. Os critérios de inclusão foram: trauma envolvendo toda a cavidade torácica em seus dois eixos, penetrante ou fechado. Os pacientes excluídos foram aqueles que tiveram tórax drenado de origem não-traumática. Os dados colhidos foram analisados através do programa Epi.Info 6.02. O trabalho foi aprovado pela direção e pelo comitê de ética do hospital.

\section{RESULTADOS}

Dos 168 pacientes, 120 eram do sexo masculino com idade entre 15 e 66 anos, média de 40 anos. As mulheres tinham idade entre 17 a 45 anos, com média de 31 anos. O trauma penetrante por arma branca foi predominante, ocorrendo em 142 casos, por arma de fogo em oito casos e em 18 o traumatismo foi do tipo fechado.

Do total de pacientes, 137 realizaram radiografia de tórax no momento da admissão. Foram excluídos oito pacientes vítimas de lesão por projétil de arma de fogo (PAF) no tórax que sofreram intervenção cirúrgica imediata e dezoito que tiveram que ser submetidos à drenagem torácica de emergência devido a hemo ou pneumotórax grave. Nos cinco pacientes restantes, a gravidade foi diagnosticada clinicamente pelas próprias condições de hipóxia apresentadas pelos pacientes.

Dos 142 traumatizados de tórax por arma branca, dez foram submetidos à toracotomia de urgência(Tabela 1), dos quais quatro sofreram ferimento cardíaco, tendo sido o ventrículo direito atingido em todas as ocasiões. Em quatro

1.Cirurgião Geral, Hospital Djalma Marques.

Recebido em 10/08/2004

Aceito para publicação em 17/01/2005

Trabalho realizado no Hospital Djalma Marques, São Luís-MA. 
pacientes, houve lesão de vasos pulmonares, e em um deles foi necessário realizar uma lobectomia. Outro teve lesão de pericárdio, que foi devidamente explorado e deixado aberto. Um paciente foi pneumonectomizado, devido à lesão do hilo pulmonar e faleceu durante o período intra-operatório. Outros dois pacientes faleceram no período pós-operatório, um devido à insuficiência renal aguda e o outro por hemorragia digestiva alta.

Um paciente apresentou fístula broncopleural após drenagem torácica por ferimento por arma branca, que teve que ser corrigida cirurgicamente por minitoracotomia à direita e aerostasia por sutura seletiva do brônquio.

Todos os pacientes com o tórax lesado por PAF(projétil de arma de fogo) tiveram resolução com drenagem torácica fechada simples, sem toracotomia. Da mesma forma, dos dezoito pacientes com traumatismo torácico fechado, todos tiveram resolução com drenagem torácica.

Os 168 pacientes fizeram uso de cefalosporina de $1^{\mathrm{a}}$ geração $^{7,8}$, como prevenção de infecção na cavidade pleural. Os drenos torácicos foram retirados entre três a sete dias baseados em dois critérios fundamentais: baixo débito e expansibilidade pulmonar satisfatória à teleradiografia de tórax.

O período de internação variou de três a 21 dias, com média de 12 dias.

\section{DISCUSSÃo}

O traumatismo torácico exige muitas vezes, diagnóstico rápido e intervenção imediata. Assim, é necessário que o cirurgião de emergência avalie rapidamente as condições clínicas do paciente, dando ênfase à oxigenação, à presença ou não de choque hipovolêmico e uma vez tendo drenado o hemitórax atingido, observar se a drenagem torácica está sendo eficaz e se não há lesões orgânicas associadas que mereçam tratamento assim que haja estabilização respiratória e circulatória ${ }^{5}$.

Os doentes com trauma fechado ou aberto que se encontravam estáveis, tiveram documentação radiológica antes de qualquer intervenção e os mais graves, hipóxicos ou chocados foram encaminhados diretamente ao centro cirúrgico para tratamento imediato. Os feridos por PAF, além da drenagem torácica, foram investigados com ultra-sonografia ou submetidos à laparotomia de urgência devido à presença de lesões associadas.
Quanto ao índice de toracotomias de urgência, alguns autores advogam cinco indicações clássicas após as medidas iniciais: a) tamponamento cardíaco, b)toracotomia traumática, c) lesão de traquéia, brônquio ou esôfago, d) hemotórax maciço e e) lesão de grandes vasos ${ }^{1,5}$. Na nossa casuística, as toracotomias ocorreram devido a: ferimento cardíaco (quatro), lesão de vasos do pedículo pulmonar (quatro), lesão de saco pericárdico (um) e do hilo pulmonar(um) envolvendo a tríade broncovascular pulmonar, o que está de acordo com a literatura mundial. Todos os pacientes com ferimento cardíaco tiveram a lesão suturada com fio de polipropileno. A mesma conduta foi utilizada com vasos pulmonares lesados, quando a sutura foi factível. Quando não, foi feita ligadura dupla do vasos com fio inabsorvível.

A paciente que teve pericardiotomia traumática foi submetida à exploração minuciosa do saco pericárdico através de incisão submamária esquerda e não tendo sido encontrado lesão cardíaca, o pericárdio foi deixado aberto para evitar qualquer possibilidade de tamponamento. Nesta paciente foi utilizada apenas um tubo $32 \mathrm{~F}$ para drenagem do espaço pleural, os demais tiveram dupla drenagem em virtude de suas lesões serem de maior magnitude.

Ainda sobre a drenagem pleural, muitos autores defendem que o período máximo para permanência do dreno na cavidade pleural seja de três a oito dias ${ }^{6-8}$, devido aos riscos de empiema. No presente estudo, os pacientes ficaram drenados por três a sete dias, e nenhum apresentou esta complicação.

Em relação aos óbitos, dois deles decorreram de complicações clínicas e um ainda no trans-operatório após pneumonectomia. É sabido que $50 \%$ dos pacientes pneumonectomizados de emergência evoluem para óbito devido à insuficiência do pulmão remanescente em manter a oxigenação corporal e também por insuficiência do ventriculo direito em suportar a pré-carga e manter uma pressão pulmonar sem que o paciente desenvolva edema de pulmão ${ }^{9}$. O paciente pneumonectomizado teve lesão de todos os elementos do hilo pulmonar direito e entrou em choque hipovolêmico rapidamente, de modo que quando realizada a toracotomia e identificada a injúria, as condições clínicas do paciente já eram críticas, o que terminou levando-o ao óbito mesmo após rápido clampeamento em massa do pedículo seguido da pneumonectomia.

Tabela 1 - Pacientes submetidos a toracotomia de urgência.

\begin{tabular}{lcll}
\hline Sexo & Idade & Tipo de Lesão & Tratamento \\
\hline Masculino & 29 & Ferimento cardíaco & Cardiorrafia \\
Masculino & 25 & Ferimento cardíaco & Cardiorrafia \\
Masculino & 33 & Ferimento cardíaco & Cardiorrafia \\
Masculino & 32 & Ferimento cardíaco & Pneumorrafia \\
Masculino & 28 & Lesão de vaso pulmonar & Pneumorrafia \\
Masculino & 28 & Lesão de vaso pulmonar & Ligadura seletiva \\
Masculino & 23 & Lesão de vaso pulmonar & Lobectomia \\
Masculino & 36 & Lesão de vaso pulmonar & Pneumonectomia \\
Masculino & 15 & Lesão do pedículo pulmonar & Exploração \\
Feminino & 21 & Lesão pericárdica &
\end{tabular}


Enfim, as feridas torácicas são responsáveis por $20 \%$ de todas as mortes por trauma e por mais de $30 \%$ das lesões traumáticas. A mortalidade dos pacientes hospitalizados com apenas uma lesão isolada do tórax varia de 4 a $8 \%$ porém aumenta para $25 \%$ quando outro órgão é atingido e para $35 \%$ quando há comprometimento de múltiplos sistemas orgâni$\cos { }^{10}$. Daí a necessidade de se ter equipes cirúrgicas muito bem treinadas para atender o politraumatizado e em especial o traumatizado de tórax.

Este estudo demonstra que a mortalidade é baixa quando as medidas iniciais são instituídas rapidamente por equipe cirúrgica bem treinada e quando o hospital é de referência para o atendimento de grandes traumatizados.

\begin{abstract}
Background: Our objective is to study thoracic trauma cases treated in a public hospital. Methods: A retrospective study of 168 cases of thoracic trauma, enphasizing the initial approach, surgical and postoperative care. Results: Of 168 patients, 120 were males with an avarage age of 35.5 years. Ten patients underwent thoracotomy as an emergency procedure: four heart wounds, four lung vessels lesions, one pericardial sac injury and one lesion of the lung hilum. The others had their clinical conditions resolved through thoracic drainage. There were two non surgical deaths and one death due to breathing failure. Conclusions: Thoracic trauma is still frequent, and the majority of cases are simple. On the othem hand some will require surgical skill and a high level support Hospital.
\end{abstract}

Key-words: Trauma, Thoracic injuries: Thorax.; Postoperative care.

\section{REFERÊNCIAS}

1. Giannini JÁ, Soldá SC, Saad Júnior R - “Trauma de tórax”. In Coimbra RSM, Soldá SC, Casaroli AA, et al. - Emergências traumáticas e não traumáticas. São Paulo. Atheneu, 2001, pp. 57-69.

2. Lo Cicero J, Mattox KL - Epidemiology of chest trauma. Surg Clin North Am. 1989, 69(1):15-19.

3. Calhoon JH, Trinkle JK - Pathophysiology of chest trauma. Chest Surg Clin North Am. 1997, 7(2):199-211.

4. American College of Surgeons. Manual de Suporte Avançado de

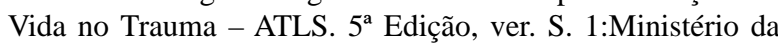
Saúde, 1993, 414 p.

5. Fragomeni LS, Pertuzzatti ML, Falleiro R, et al. -Trauma torácico: manejo clínico-cirúrgico. Med Cir, 1994, 6(1):38-42.

6. Lo Curto JJ, Tischler CD, Swan KG, et al. - Tube thoracotomy and trauma - antibiotics or not? J Trauma. 1986, 26(12):10671072.
7. Stone HH, Symbas PN, Hooper CA - Cefamandole for profilaxis against in closed tube thoracotomy. J Trauma. 1981, 21(11):975977.

8. Etoch SW, Bar-Natan MF, Miller FB, et al. - Tube thoracostomy: factors related to complications. Arch Surg. 1995, 130(5):521-525; discussion 525-526.

9. Tominaga GT, Waxman K, Scannell G, et al. - Emergency thoracotomy with lung resection following trauma. Am Surg. 1993, 59(12):834-837.

10. Silas MG, Belluzzo GR, Miguel EG, et al. - Traumatismos torácicos: análise de 231 casos. Arq Méd ABC. 1990, 13(1/ 2):19-21.

Endereço Para Correspondência:

Dr. Ricardo Cuba

Rua 11, nº 20, Quadra 108, Conj. Penalva

São Cristóvão.

São Luis - MA - 65055-000 